\title{
Zum Verhältnis von Anhaltungsverfügung nach KultGüRückG und polizei- rechtlicher Sicherstellungsverfügung (I)
}

Hessischer Verwaltungsgerichtshof, Beschluss vom 4. Juli 2011 - 8 A 1526/10.Z

1. §8 Abs. 6 KultGüRückG ist lex specialis zu §40 Nrn. 2 und 4 HSOG; ihre Anwendung kann nicht dadurch umgangen werden kann, dass auf die allgemeine Vorschrift des $\$ 40$ Nrn. 2 und 4 HSOG zurückgegriffen wird, falls die Voraussetzungen des $\$ 8$ Abs. 6 KultGüRückG nicht vorliegen.

2. Unabhängig davon, dass $\$ 8$ Abs. 6 KultGüRückG die speziellere Norm ist, kann die Sicherstellung auch nicht auf 40 Nr. 4 HSOG gestützt werden, wenn keine Anhaltspunkte dafür vorliegen, dass die Münzen zur Begehung einer Straftat oder einer Ordnungswidrigkeit verwendet werden sollen. (Leitsätze der Redaktion)

\section{Beschluss \\ - In dem Verwaltungsstreitverfahren}

des A. (...), Klägers und Zulassungsantragsgegners,

gegen

das Land Hessen, vertreten durch das Hessische Ministerium für Wissenschaft und Kunst, (...), Beklagten und Zulassungsantragsteller,

wegen Sicherstellung

hat der Hessische Verwaltungsgerichtshof -8 . Senat - (...) am 4. Juli 2011 beschlossen:

Der Antrag des Beklagten auf Zulassung der Berufung gegen das Urteil des Verwaltungsgerichts Gießen vom 6. Mai 2010 - 9 K 1661/09.GI (Anmerkung der Redaktion: in diesem Heft, S. 125 ff.) - wird abgelehnt. Der Beklagte hat die Kosten des Zulassungsantragsverfahrens zu tragen. Der Streitwert wird auch für das Zulassungsantragsverfahren auf 20.000,- $€$ festgesetzt.

\section{Gründe}

Der Antrag auf Zulassung der Berufung gegen das dem Beklagten am 25. Juni 2010 zugestellte Urteil hat keinen Erfolg. Die in der Zulassungsantragsbegründung vom 25. August 2010, bei dem Hess. VGH am selben Tage eingegangenen, geltend gemachten Berufungszulassungsgründe der ernstlichen Zweifel an der Richtigkeit des Urteils (§ 124 Abs. 2 Nr. 1 VwGO), der besonderen tatsächlichen oder rechtlichen Schwierigkeit der Rechtssache ( $\$ 124$ Abs. 2 Nr. 2 VwGO) und der grundsätzlichen Bedeutung der Rechtssache ( $\$ 124$ Abs. 2 Nr. 3 VwGO) liegen nicht vor beziehungsweise sind nicht hinreichend dargetan.

Ernstliche Zweifel an der Richtigkeit des Urteils im Sinne des § 124 Abs. 2 Nr. 1 VwGO sind dann begründet, wenn ein einzelner tragender Rechtssatz oder eine erhebliche Tatsachenfeststellung mit schlüssigen Argumenten infrage gestellt wird (vgl. BVerfG Beschluss vom 26. März 2007 -1 BvR 2228/02 - juris, Rn. 25, mwN). Diese Voraussetzung ist nicht gegeben. Das Urteil des Verwaltungsgerichts
Gießen ist im Ergebnis nicht zu beanstanden, wobei dahinstehen kann, ob die Sicherstellungsverfügung des Beklagten vom 15. Juli 2009 wegen fehlender Zuständigkeit des Beklagten bereits formell rechtswidrig ist, denn die Verfügung ist jedenfalls materiell rechtswidrig. Sie entspricht nicht den Voraussetzungen des § 8 Abs. 6 des Kulturgüterrückgabegesetzes (KultGüRückG). Danach ist das angehaltene Kulturgut nach Maßgabe landesrechtlicher Vorschriften sicherzustellen, sofern zu befürchten ist, dass seine Rückgabe an den ersuchenden Staat verhindert werden soll oder dass es Schaden erleidet. Eine Sicherstellung nach § 8 Abs. 6 KultGüRückG erfordert zunächst das Vorliegen einer Anhaltung im Sinne des $\S$ 8 Abs. 2 KultGüRückG. Daran fehlt es bereits, denn es ist auch im Beschwerdeverfahren nicht vorgetragen worden, dass eine Anhaltungsverfügung hinsichtlich der sichergestellten Münzen des Klägers ergangen wäre. Vielmehr trägt der Beklagte auf S. 3 der Zulassungsantragsbegründung sogar vor, er habe nicht im Rahmen des $\S 8$ Abs. 6 KultGüRückG gehandelt, denn dafür sei eine Anhaltungsverfügung erforderlich. Auch die weiteren Voraussetzungen des $\S 8$ Abs. 6 KultGüRückG liegen nicht vor, denn es fehlt bereits an dem „ersuchenden Staat " in diesem Sinne, weil ein entsprechendes Ersuchen nicht vorliegt. Der Beklagte hat auch keine sichere Kenntnis darüber, welcher Staat für ein entsprechendes Ersuchen in Frage kommen könnte. Schließlich ist auch nicht dargelegt, inwieweit die sichergestellten Münzen Schaden erleiden sollten.

Soweit der Beklagte der Ansicht ist, die Sicherstellung habe unabhängig von $\S 8$ Abs. 6 KultGüRückG aufgrund der Vorschrift des $\S 40 \mathrm{Nr}$. 2 und 4 HSOG erfolgen dürfen, kann dem nicht gefolgt werden. § 8 Abs. 6 KultGüRückG ist insoweit die speziellere Norm, die nicht dadurch umgangen werden kann, dass auf die allgemeine Vorschrift des $\S 40 \mathrm{Nr}$. 2 und 4 HSOG zurückgegriffen wird, falls die Voraussetzungen des $\S 8$ Abs. 6 KultGüRückG nicht vorliegen. Kulturgüter im Sinne des Kulturgüterrückgabegesetzes unterliegen nicht dem gleichen Schutz wie Sachen, die gemäß § 40 Nr. 2 HSOG geschützt werden sollen. Dies ergibt sich bereits aus den Fristen des $\S 6$ KultGüRückG, innerhalb derer ein Rückgabeersuchen eines anderen Staates wegen eines in das Bundesgebiet verbrachten Gegenstandes erfolgen muss, um die Rückgabe erfolgreich verlangen zu können. Mit der Anhaltung gemäß § 8 Abs. 2 KultGüRückG steht der zuständigen Behörde außerdem auch eine spezielle Maßnahme zur Verfügung, mit der sie den Fällen begegnen kann, in denen der dringende Verdacht besteht, dass Kulturgut unrechtmäßig in das Bundesgebiet verbracht wor- 
den und einem anderen Staat zurückzugeben ist. Es besteht im Übrigen keinerlei Anhaltspunkt dafür, dass der Kläger nicht Eigentümer der rechtswidrig sichergestellten Münzen geworden ist.

Die Sicherstellung kann auch nicht auf $\S 40$ Nr. 4 HSOG gestützt werden, denn unabhängig davon, dass $\S 8$ Abs. 6 KultGüRückG die speziellere Norm ist, liegen auch, wie das Verwaltungsgericht zutreffend ausführt, keine Anhaltspunkte dafür vor, dass die Münzen zur Begehung einer Straftat oder einer Ordnungswidrigkeit verwendet werden sollen.

Der Zulassungsgrund der besonderen tatsächlichen und rechtlichen Schwierigkeiten der Rechtssache (§ 124 Abs. 2 Nr. 2 VwGO) erfordert überdurchschnittliche, das normale Maß nicht unerheblich überschreitende Schwierigkeiten (vgl. dazu Kopp/ Schenke VwGO, 15. Aufl. 2007, § 124, Rn. 9). Zu überprüfen war die Frage, ob die Beklagte die Sicherstellung der Münzen auf der Grundlage des $\S 40$ Nr. 2 und 4 HSOG vornehmen durfte. Wie die Ausführungen im Rahmen der Prüfung des § 124 Abs. 2 Nr.1 VwGO ergeben haben, lässt sich diese Frage anhand des Gesetzes beantworten und wirft keine überdurchschnittlichen Schwierigkeiten auf.
Auch der Zulassungsgrund der grundsätzlichen Bedeutunq der Rechtssache im Sinne des § 124 Abs. 2 Nr. 3 VwGO ist nicht hinreichend dargetan. Zur Darlegung dieses Zulassungsgrundes ist es erforderlich, eine bestimmte, bisher noch ungeklärte und für die Entscheidung des Oberverwaltungsgerichts/Verwaltungsgerichtshofs erhebliche Rechtsfrage zu formulieren und anzugeben, worin die allgemeine, über den Einzelfall hinausgehende Bedeutung dieser Rechtsfrage bestehen soll (vgl. Hess. VGH Beschluss vom 17. Juli 1998 - 8 ZU 2071/98 -, unter Hinweis auf BVerwG Beschluss vom 19. August 1997 - 7 B 261/97 = NJW 1997, 3328). Eine solche Darlegung lässt sich jedoch den Ausführungen des Beklagten nicht entnehmen. Die von dem Beklagten formulierte Frage lässt sich anhand der gesetzlichen Vorschriften beantworten.

Die Kostenentscheidung beruht auf $\S 154$ Abs. 2 VwGO, die Streitwertfestsetzung folgt der erstinstanzlichen Wertfestsetzung, zumal der Beklagte dagegen keine Einwendungen erhoben hat.

Dieser Beschluss ist unanfechtbar (§ 152 Abs. 1 VwGO: $\S 66$ Abs. 3 Satz 3 GKG iVm $\S 68$ Abs. 1 Satz 5 GKG). (Entscheidung von der Redaktion bearbeitet.)

\section{Zum Verhältnis von Anhaltungsverfügung nach KultGüRückG und polizei- rechtlicher Sicherstellungsverfügung (II)}

VG Gießen, Urteil vom 6. Mai 2010 - 9 K 1661/09.GI

\section{1. § 8 Abs. 1, 6 KultGüRückG geht aufgrund der besonderen Ausformung des Eigentumsschutzes als lex specialis} der Vorschrift des $\$ 40$ Nr. 2 HSOG vor.

2. Nach § 85 Abs. 1 Nr. 1 HSOG können die fachlich zuständigen Ministerien zwar als Landesordnungsbehörden und damit als allgemeine Ordnungsbehörden handeln; dies jedoch nur bei einem Handeln im Rahmen der fachlichen Zuständigkeit, die sich indes über die Vorschriften des Kulturgüterrückgabegesetzes definiert und für die Sicherstellung von Kulturgütern die Spezialregelung des § 8 Abs. 1, 6 KultGüRückG vorsieht; eine darüber hinausgehende Zuständigkeit zur präventiven Bekämpfung von Straftaten und Ordnungswidrigkeiten durch Sicherstellungsmaßnahmen gemäß $\$ 40$ Nr. 4 HSOG ist nicht eröffnet. Darüber hinaus zählt der Bereich des Kulturgüterschutzes nach dem Kulturgüterrückgabegesetz nicht zu den, den allgemeinen Ordnungsbehörden gemäß § 1 HSOG-DVO, § 89 Abs. 1 HSOG zugewiesenen Aufgabenbereichen.

3. Die Umdeutung einer angefochtenen Sicherungsverfügung in eine entsprechende Sicherungsverfügung nach Maßgabe des Kulturgüterrückgabegesetzes verbietet sich, wenn die Voraussetzungen für eine Sicherstellung nach § 8 Abs. 6 Kult GüRückG offensichtlich nicht vorliegen. (Leitsätze der Redaktion)

\section{Tenor}

- Der Bescheid des Hessischen Ministeriums für Wissenschaft und Kunst vom 15. Juli 2009 wird aufgehoben.

Die Kosten des Verfahrens werden dem Beklagten auferlegt.

Das Urteil ist hinsichtlich der außergerichtlichen Kosten des Klägers vorläufig vollstreckbar. Der Kostenschuldner darf die Vollstreckung durch Sicherheitsleistung oder Hinterlegung nach Maßgabe der Kostenfestsetzung abwenden, falls nicht der Gläubiger vor der Vollstreckung Sicherheit in derselben Höhe leistet.

\section{Tatbestand}

Die Beteiligten streiten über die Sicherstellung von 821 Münzen durch das beklagte Land. Im Zuge von Ermittlungen des Polizeipräsidiums Mittelhessen, Kriminaldirektion Gießen, gegen einen Herrn (...) wegen des Verdachts der Unterschlagung und 